\title{
Latanoprost administered once daily caused a maintained reduction of intraocular pressure in glaucoma patients treated concomitantly with timolol
}

\author{
Albert Alm, Ingmar Widengård, Daniel Kjellgren, Mats Söderström, Björn Friström, \\ Anders Heijl, Johan Stjerschantz
}

\begin{abstract}
The long term effects of two dose regimens of latanoprost (PhXA41) administered to eyes concomitantly treated with timolol which had not adequately been controlled by timolol alone were compared. A total of 50 patients, 17 with primary open angle glaucoma and 33 with capsular glaucoma, were recruited from five clinics. All had glaucomatous visual field defects and an intraocular pressure (IOP) of at least $22 \mathrm{~mm} \mathbf{~ H g}$ despite treatment with $0.5 \%$ timolol twice daily. Patients were randomised to two treatment groups. In one group $\mathbf{0 . 0 0 6 \%}$ latanoprost was given twice daily, in the other group placebo was given at 8 am and latanoprost at $8 \mathrm{pm}$ for 3 months, with concomitant timolol treatment in both groups. Average daytime IOP (mean (SD)) at baseline (on timolol alone) and after 4 and 12 weeks' treatment was $24.8(3.6), 16.8(4.3)$, and $15.7(2.4)$ $\mathrm{mm} \mathrm{Hg}$ respectively with once daily application of latanoprost and 24.9 (2.9), $18 \cdot 1$ (3.0), and 18.0 (3.6) $\mathrm{mm} \mathrm{Hg}$ respectively with latanoprost twice daily. No clinically significant side effects were observed during treatment. Latanoprost causes a marked and sustained IOP reduction in eyes which are also being treated with timolol. Latanoprost given once daily is at least as effective and probably superior to a twice daily dose regimen

(BrF Ophthalmol 1995; 79: 12-16)
\end{abstract}

Several previous studies have demonstrated that the phenyl substituted prostaglandin $F_{2 \alpha}$ analogue, 13,14-dihydro-17-phenyl-18,19, 20-trinor-prostaglandin $\quad \mathrm{F}_{2 \alpha}$-isopropylester (latanoprost, PhXA41) and its epimeric mixture $\mathrm{PhXA} 34$ reduce significantly the intraocular pressure (IOP) in normal, ocular hypertensive, or glaucomatous eyes. ${ }^{1-8}$ The present study was undertaken to obtain more information on dose regimen, long term effect, and additivity to a $\beta$ adrenergic antagonist, timolol.

Latanoprost has a long duration of effect on IOP, but whether it should be administered once of twice daily is unclear. In two dose finding studies with the epimeric mixture $\mathrm{PhXA34}$ a duration of at least 24 hours was observed but the effect 24 hours after the dose was less pronounced than that seen at 12 hours after the dose. ${ }^{2}$ Such an attenuation of the effect was not observed in a study on hospitalised patients treated with latanoprost, ${ }^{7}$ and in one dose regimen study administration of $0.006 \%$ latanoprost once daily was at least as effective as twice daily. ${ }^{4}$
In a first dose finding study with twice daily administrations of latanoprost, ocular hypertensive eyes were treated for 4 weeks. ${ }^{5}$ There was no significant difference between the three concentrations of latanoprost eye drops used; $0.0035 \%$, $0.006 \%$, and $0.0115 \%$, and all were significantly better than placebo. The initial response, on the second day of treatment, was good with a 31$38 \%$ reduction of the IOP, but after 1 week of treatment there was some diminution of effect and after 4 weeks the IOP reduction was between 19 and $22 \%$ for the three concentrations of latanoprost used. A partial diminution in the IOP effect was also observed by Camras et al after 5 days of treatment twice daily with $0.01 \%$ latanoprost, but not with $0.003 \% .{ }^{3}$ Friström and Nilsson also noted some reduction of the IOP effect with $0.006 \%$ latanoprost given twice daily for 1 week, similar to that observed for $2 \%$ pilocarpine administered three times daily. They also found that the effect on IOP of latanoprost and pilocarpine was at least partially additive. ${ }^{9}$ Both drugs act on outflow; latanoprost has no effect on aqueous flow. ${ }^{168}$ Thus one would expect latanoprost and an aqueous flow suppressor to be a better combination. The effect on IOP of prostaglandin $F_{2 \alpha}$-isopropylester $\left(\mathrm{PGF}_{2 \alpha}-\mathrm{IE}\right)$ has previously been found to be additive to that of timolol ${ }^{1011}$ and a direct comparison of the additivity of latanoprost to timolol $0.5 \%$ twice daily and pilocarpine $2 \%$ three times daily suggested that pretreatment with pilocarpine reduced the effect of latanoprost on IOP. ${ }^{12}$

The present study was designed to evaluate the effect of latanoprost administered either once or twice daily in addition to timolol. Treatment was given for 3 months to be able to detect any long term diminution in the effect of the drug on IOP.

\section{Patients and methods}

\section{SUBJECT SELECTION}

The study was performed as a five centre, randomised, parallel, double masked study of latanoprost, $0.006 \%$, given either once, in the evening, or twice daily for 12 weeks. Patients of either sex over the age of 60 , with primary open angle glaucoma (POAG) or capsular glaucoma, in whom the IOP was not adequately controlled despite $0.5 \%$ timolol twice daily were included. Inadequate IOP control was defined as an IOP of at least $22 \mathrm{~mm} \mathrm{Hg}$ on two occasions taken at an interval of at least 1 hour at the pre-inclusion examination. Ten patients were recruited from each centre, five per treatment group. Patients 
Table 1 Examination schedule

\begin{tabular}{|c|c|c|c|c|c|c|c|c|c|c|c|c|c|c|}
\hline \multirow[b]{2}{*}{ Investigational event } & \multirow{2}{*}{$\begin{array}{l}\text { Pre-inclusion } \\
\text { within } 1 \text { month }\end{array}$} & \multicolumn{3}{|c|}{ Day 0} & \multirow{2}{*}{$\frac{\text { Day } 14}{4}$} & \multicolumn{3}{|c|}{ Day 28} & \multirow{2}{*}{$\begin{array}{l}\text { Week } 6 \\
4 \text { pm }\end{array}$} & \multirow{2}{*}{$\begin{array}{l}\text { Week } 8 \\
4 \mathrm{pm}\end{array}$} & \multirow{2}{*}{$\begin{array}{l}\text { Week } 10 \\
4 \mathrm{pm}\end{array}$} & \multicolumn{3}{|c|}{ Week 12} \\
\hline & & 8 & 12 & 4 & & 8 & 12 & 4 & & & & 8 & 12 & 4 \\
\hline Ocular examination & 夫 & & & & & & & & & & & & * & \\
\hline $\begin{array}{l}\text { Symptomatology } \\
\text { Visual acuity }\end{array}$ & $\star$ & $\star$ & $\star$ & $\star$ & 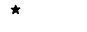 & $\star$ & $\star$ & ^ & $\star$ & $\star$ & $\star$ & $\star$ & * & * \\
\hline $\begin{array}{l}\text { Visual acuity } \\
\text { Biomicroscopy }\end{array}$ & $\star$ & $\star$ & & & & ^ & & & & & & $\star$ & & \\
\hline $\begin{array}{l}\text { Biomicroscopy } \\
\text { Photography }\end{array}$ & * & * & * & $\star$ & $\star$ & $\star$ & * & * & $\star$ & $\star$ & 夫 & * & $\star$ & ネ \\
\hline $\begin{array}{l}\text { Photography } \\
\text { Hyperaemia grading }\end{array}$ & 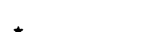 & $\star$ & & ^ & ఓ & $\star$ & $\star$ & $\star \star$ & ᄎ & ᄎ & ઔ & & & * \\
\hline $\begin{array}{l}\text { Hyperaemia grading } \\
\text { IOP }\end{array}$ & $\stackrel{\star}{\star}$ & $\stackrel{\star}{\star}$ & $\stackrel{\star}{\star}$ & $\star$ & * & $\star$ & * & 夫 & $\star$ & $\star$ & $\star$ & & & $\star$ \\
\hline
\end{tabular}

with a history of severe ocular trauma, severe ocular inflammation within the past 3 months, or intraocular surgery within the last 6 months were excluded. Exclusion criteria also included known contraindications to $\beta$ adrenergic blockers, a history of acute angle closure glaucoma, a history of significant dry eyes, and wearing contact lenses. The study protocols were reviewed and approved by the National Board of Health and Welfare and by the appropriate local review boards. Written informed consent was obtained from all subjects.

\section{EXAMINATION SCHEDULE AND PROCEDURES}

A pre-inclusion examination was performed within 1 month before the study. We obtained a medical and ophthalmic history including information on ocular symptoms. In addition to IOP determinations the initial examination included measurement of refractive error and Snellen visual acuity, a slit-lamp evaluation of the anterior segment, ophthalmoscopic examination of the optic nerve, and a visual field if one had not been performed within the last 6 months.

The examination schedule is presented in Table 1. The patients visited the clinic three times on the baseline day and 4 and 12 weeks after initiation of treatment but only once daily for examinations after $2,6,8$, and 10 weeks' treatment. The degree of conjunctival hyperaemia was graded in the treated eye by comparison with four standard photographs corresponding to no hyperaemia, mild, moderate, and severe hyperaemia respectively. A photograph was taken of the anterior segment of the eye which was later evaluated in a masked manner.

The patients were randomised to two treatment groups. Each patient was provided with one bottle of $0.5 \%$ timolol (Blocadren, MSD) and two identical bottles, one labelled morning and one evening. For group $A$ both bottles contained $0.006 \%$ latanoprost, for group B the morning bottle contained vehicle and the evening bottle $0.006 \%$ latanoprost. Timolol was always administered first with 5 minutes between eye drops. The first dose of latanoprost or vehicle was given in the evening, about $8 \mathrm{pm}$, of the baseline day (day 0 ). Patients were instructed to administer the drops in the morning at $8 \mathrm{am}$ and in the evening at $8 \mathrm{pm}$ throughout the study. Twenty patients were treated with timolol in both eyes before entering the study. These patients continued with timolol treatment in the fellow eye also during the study.

One patient had been on timolol for only 2 weeks before entering the study, six patients between 1 and 4 months, and the remaining 43 patients, at least 6 months.
STATISTICAL ANALYSIS AND EVALUATION OF EFFECT

Diurnal IOP was defined as the mean IOP over the day based on the values obtained at $8 \mathrm{am}, 12$ noon, and $4 \mathrm{pm}$. Owing to differences in baseline IOP between patients, baseline IOP was used as a covariate in statistical analysis of the IOP reduction. The difference in IOP reduction between treatment groups at week 12 at 4 pm was analysed by two way analysis of covariance with treatment group and centre as factors and baseline IOP as covariate. Maximal hyperaemia was defined as the highest score from the three measurements during the day. Change in maximal hyperaemia at week 12 within treatment groups was tested with the sign test. Comparison of change in maximal hyperaemia at week 12 between treatment groups was performed with Wilcoxon rank sum test. The $\chi^{2}$ test was used to compare the number of patients who responded positively to latanoprost in the two groups.

\section{Results}

Of the 50 patients who entered 48 were able to complete the study. The two patients who withdrew were in the group which administered latanoprost once daily. One patient developed a keratitis after 4 weeks' treatment and one withdrew after 6 weeks and 4 days' treatment owing to difficulties in distinguishing between the dropper bottles.

Oral $\beta$ blocking agents were used by one patient in the twice daily group compared with seven patients in the once daily group. Otherwise there were no major differences between treatment groups with respect to demographic or clinical data (Table 2).

The mean diurnal IOPs at baseline and weeks 4 and 12 for the two groups are presented in Table 3, and the IOPs measured at $4 \mathrm{pm}$ at baseline and at 2 week intervals for 12 weeks are shown in Figure 1. After 4 weeks' treatment the mean diurnal IOP was reduced by $8.0 \mathrm{~mm} \mathrm{Hg}$ in

Table 2 Demographic and clinical characteristics

\begin{tabular}{lccc}
\hline & $\begin{array}{c}\text { Once } \\
\text { daily }\end{array}$ & $\begin{array}{l}\text { Twice } \\
\text { daily }\end{array}$ & All \\
\hline Age & & & \\
Mean & $74 \cdot 0$ & $72 \cdot 6$ & $73 \cdot 3$ \\
$\quad$ Range & $60-84$ & $63-87$ & $60-87$ \\
Sex (M/F) & $6 / 19$ & $9 / 16$ & $15 / 35$ \\
Iris colour & & & \\
$\quad$ Blue/green & 21 & 20 & 41 \\
$\quad$ Brown & 3 & 1 & 4 \\
$\quad$ Grey & 1 & 4 & 5 \\
Diagnosis & 8 & 9 & 17 \\
$\quad$ POAG & 17 & 16 & 33 \\
$\quad$ Capsular glaucoma & 11 & 8 & 19 \\
Family history of OH or glaucoma & 1 & 7 & 8 \\
Use of oral $\beta$ blockers & & & \\
\hline
\end{tabular}

POAG = primary open angle glaucoma. $\mathrm{OH}=$ ocular hypertension. 
Table 3 Mean (SD) diurnal IOP ( $m m$ Hg) at baseline, week 4 , and week 12

\begin{tabular}{llll}
\hline Treatment & Baseline & Week 4 & Week 12 \\
\hline Once daily & $24 \cdot 8(3 \cdot 6)$ & $16 \cdot 8(4 \cdot 3)$ & $15 \cdot 7(2 \cdot 4)$ \\
Twice daily & $24.9(2 \cdot 9)$ & $18 \cdot 1(3 \cdot 0)$ & $18 \cdot 0(3.6)$ \\
p Values for test between & & $0 \cdot 15$ & $<0 \cdot 01$ \\
$\quad$ treatments & & 0.15 & \\
\hline
\end{tabular}

the once daily group and by $6.8 \mathrm{~mm} \mathrm{Hg}$ in the twice daily group which corresponds to 32 and $27 \%$ of baseline diurnal IOP, respectively. After 12 weeks' treatment the corresponding figures were $9.1 \mathrm{~mm} \mathrm{Hg} \mathrm{(37 \% )} \mathrm{and} 6.9 \mathrm{~mm} \mathrm{Hg} \mathrm{(28 \% )}$ respectively, with a statistically significant difference in IOP between the two groups $(\mathrm{p}<0.01)$.

A post hoc analysis was made to compare the clinical efficiency of the two dose regimens. Thus patients were judged to show a good response to latanoprost if they fulfilled one or both of the following definitions: (1) No recorded IOP over $21 \mathrm{~mm} \mathrm{Hg}$ for any of the $10 \mathrm{IOP}$ measurements during treatment in the follow up. (2) An IOP reduction of at least $15 \%$ compared with baseline on all 10 examinations during treatment.

In the once daily group 23 of 25 patients never had an IOP measurement over $21 \mathrm{~mm} \mathrm{Hg}$ compared with only 10 of 25 patients in the twice daily group. This difference was statistically significant $\left(p=0.0001, \chi^{2}\right)$. Of 25 patients treated with latanoprost once daily 21 had an IOP reduction of at least $15 \%$ on all subsequent IOP determinations compared with only eight of 25 in the twice daily group $\left(p=0.0002, \chi^{2}\right)$.

A slight but statistically significant contralateral IOP reduction was recorded in the eyes which had not been treated with latanoprost. Comparison of the $4 \mathrm{pm}$ values at baseline and week 12 revealed a $0.7 \mathrm{~mm} \mathrm{Hg}$ IOP reduction from a baseline value of $18.7 \mathrm{~mm} \mathrm{Hg}$ in the once daily group $(\mathrm{p}=0.04)$ and a $1.1 \mathrm{~mm} \mathrm{Hg}$ IOP reduction from a baseline of $19.8 \mathrm{~mm} \mathrm{Hg}(p=$ 0.03 ) in the twice daily group. An analysis of the IOP change in the subgroups of patients treated with timolol also in the contralateral eye showed for corresponding time points a change from 18.5 to $17.8 \mathrm{~mm} \mathrm{Hg}$ in the once daily group $(\mathrm{n}=$ 12) and from 21.3 to $19.8 \mathrm{~mm} \mathrm{Hg}$ in the twice daily group $(n=8)$. These changes were not statistically significant.

The maximal grades of hyperaemia observed

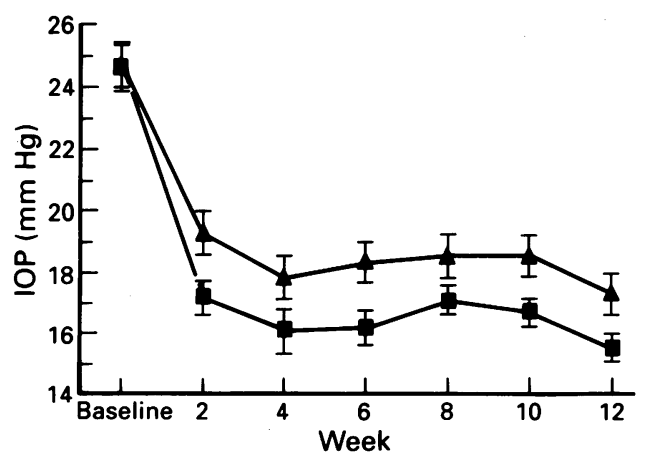

Figure 1 Mean intraocular pressure (IOP) (SEM) at $4 \mathrm{pm}$ on baseline and at 2 week intervals during treatment for 12 weeks. Both groups received $0.5 \%$ timolol twice daily at baseline and throughout the study. After the baseline measurements $0.006 \%$ latanoprost was added once daily ( or twice daily ( $\mathbf{\Delta}$.

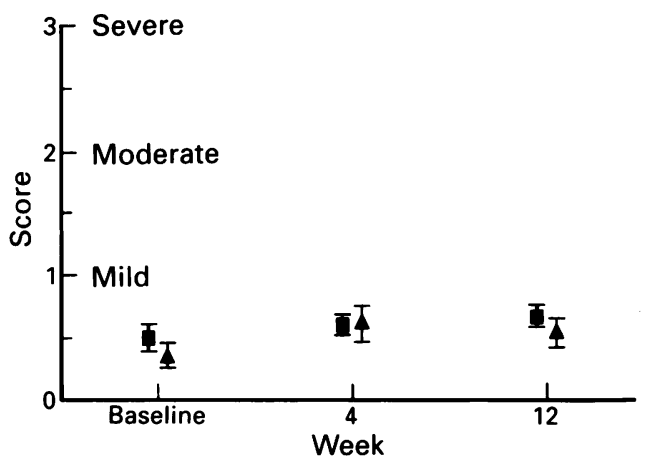

Figure 2 Maximum hyperaemia scores on baseline day and after 4 and 12 weeks' on 0.006\% latanoprost once daily ( $\square$ ) or twice daily ( $\mathbf{\Delta})$. (Means (SEM).)

during the study are presented in Figure 2. A slight trend towards increased hyperaemia in latanoprost treated eyes was observed but the average hyperaemia after 4 and 12 weeks of treatment was between none and mild in both groups. Individual variations were seen but no patient discontinued the study because of unacceptable hyperaemia. At baseline four of 25 in each group had a conjunctival injection graded as at least a mild hyperaemia. After 4 and 12 weeks of treatment this increased to seven to eight patients per group, but there was no increase in the number of patients judged to have at least a moderate hyperaemia. Two such patients were seen in the once daily group at baseline but in this group all patients were judged to have less than moderate hyperaemia after 4 and 12 weeks' treatment. In the twice daily group there was one patient with moderate hyperaemia at baseline and two patients with moderate to severe hyperaemia after 4 and 12 weeks' treatment.

In the once daily group there was one report of a mild stiffness in the study eye at baseline and one report of moderate foreign body sensation in the study eye after 4 weeks' treatment. In the twice daily group mild ocular symptoms were recorded for one eye at baseline, two eyes after 2 and 4 weeks' treatment respectively, and in one eye after 8 weeks' treatment. Mild ocular symptoms in both eyes or the fellow eye only were reported by between one and four of the 50 patients on the various examination days.

No flare was seen in any patient on any examination apart from fluorescein flare after IOP measurements. On three occasions in three different patients one cell was observed in the anterior chamber, two patients at the baseline examination and one on once daily latanoprost for 10 weeks. Photographs of the anterior segment were taken for documentation of iris colour because studies on cynomolgus monkeys have shown increased iris pigmentation with long term treatment (unpublished observation). Masked observation of the photographs of the anterior segment revealed no changes in iris pigmentation throughout the study. There were no changes in visual acuity or refraction.

\section{Discussion}

This study was designed to compare two dose regimens of latanoprost administered in addition 
to timolol. Latanoprost was given to patients with inadequate IOP control, and thus placebo was not used and timolol was not washed out before adding latanoprost. Most patients had been on timolol for at least 6 months and some were taking oral $\beta$ blockers. Consequently, the effect on IOP of timolol alone at the start of the study is not known. Comparison of the two dose regimens can be made but it is not possible to calculate the combined effect of the two drugs. The results, however, are clear; latanoprost causes a marked and significant reduction of IOP in patients who are using timolol, which has been previously shown for $\mathrm{PGF}_{2 \alpha}$-IE. ${ }^{10} 11$ The magnitude of the effect in the present study is better than in a previous study where $0.006 \%$ latanoprost was given twice daily for 1 month in otherwise untreated eyes. ${ }^{5}$ The results support the assumption that because of their different mechanisms of action the effects of timolol and latanoprost are completely additive. ${ }^{6}$

The present study also demonstrates that $0.006 \%$ latanoprost given once daily seems to be superior to twice daily administrations. Nagasubramanian et al made the same observation after 2 weeks of treatment with the same two dose regimens. ${ }^{4}$ As the results of two independent studies are the same it is unlikely that this is due to chance variation. Alternative explanations for this unusual response should be considered such as a dual effect of latanoprost on aqueous humour dynamics or the development of a moderate degree of receptor tolerance.

Latanoprost was administered in the evening to the once daily group and IOP was determined 12-20 hours later, whereas IOP determinations were made $4-8$ hours after the dose in the twice daily group. Thus one obvious possibility is that latanoprost affects aqueous humour dynamics in more than one way, a short duration effect - less than 12 hours - which would increase IOP and a long duration IOP reducing effect. In early studies with $\mathrm{PGF}_{2 \alpha}$-IE an initial increase in IOP was observed, presumably due to intraocular vasodilatation. ${ }^{13}$ An intraocular vasodilatation and an increased episcleral venous pressure lasting for 8 hours is, however, an unlikely explanation to the difference between the two dose regimens. Latanoprost, unlike $\mathrm{PGF}_{2 \alpha}$-IE, has only a slight effect on intraocular blood flow. ${ }^{14}$ No dual effect on aqueous outflow has been observed for either $\mathrm{PGF}_{2 \alpha}$ or latanoprost in monkeys; both increase uveoscleral flow without measurable effect on conventional outflow. ${ }^{14-17} \mathrm{~A}$ short duration increase of aqueous flow could explain the observed results, but previous studies both using $\mathrm{PGF}_{2 \alpha}$-IE and latanoprost have failed to demonstrate any effect on aqueous flow in the human eye..$^{681318}$ In those studies latanoprost was given alone and in the present study latanoprost was added to eyes with timolol suppressed aqueous flow, and the effect of latanoprost on aqueous flow in timolol treated eyes has not been determined. However, an increase of suppressed aqueous flow by latanoprost cannot explain the observation that the same difference between the two dose regimens was found in eyes not treated with timolol, ${ }^{4}$ and Ziai $e t$ al found that latanoprost had no effect on non-stimulated aqueous flow at night. ${ }^{8}$ Still, none of these studies were designed to detect a small change in aqueous flow and the possibility that latanoprost causes an increase of aqueous flow of, for example, $10 \%$ for 8 to 12 hours cannot be excluded.

The other possibility, is a development of a moderate degree of receptor tolerance. One argument in favour of this explanation is that the difference between the two dose regimens is not seen until after a few days of treatment. In fact, Nagasubramanian et al found that $0.006 \%$ latanoprost given twice daily was significantly superior to the same dose given once daily on the second day of the study but significantly inferior after 14 days of treatment. ${ }^{4}$ Thus, the observed phenomenon that latanoprost given once daily seems to be superior to the same dose given twice daily may be based on development of some degree of receptor tolerance. Continued treatment after the first 2 weeks does not result in further loss of effect.

In the present study a small but statistically significant reduction in IOP also occurred in the contralateral eye. A drug related effect in the fellow eye cannot be expected with latanoprost as the dose given is very small and latanoprost is rapidly metabolised with a half life in plasma of only about 10 minutes. ${ }^{19}$ Some of the fellow eyes were being treated with timolol, but these eyes did not differ from untreated fellow eyes with respect to change in IOP during the study. Improved compliance during the study cannot explain the contralateral effect which was small and of no clinical significance. It perhaps is explained by a regression towards the mean since a lower limit for IOP was part of the inclusion criteria and the IOP of the two eyes tend to vary in concert. ${ }^{20}$

Side effects were minimal in the present study. One patient interrupted the study owing to a keratitis that was judged as non-drug related. Flare was not observed in either group, and previous studies with various techniques have found no significant effect of latanoprost on the blood-aqueous barrier. ${ }^{8} \mathrm{~A}$ slight increase in conjunctival injection was noted, but was not clinically significant. There was no increase in ocular symptoms and no change in visual acuity. or refraction. Thus there is a large difference in side effects between the phenyl substituted analogue latanoprost and its mother compound $\mathrm{PGF}_{2} \alpha-\mathrm{IE}$, which caused marked conjunctival hyperaemia and ocular irritation in large doses. ${ }^{13}$ In conclusion $0.006 \%$ latanoprost given once daily causes marked reduction of the IOP in patients being treated with timolol concomitantly, and administration once daily is not only adequate but probably superior to twice daily. Thus these results support the view that latanoprost may become a valuable addition to the treatment of glaucoma.

AA is a consultant to Pharmacia Ophthalmics AB. Johan Stjernschantz is employed by Pharmacia Ophthalmics AB. The authors do not have commercial or proprietary interest in latanoprost eye drops. The results have been presented in part at the AAO meeting in Dallas, Texas, 8-12 November 1992, and at the ARVO meeting in Sarasota, Florida, 2-7 May 1993.

Financial support was provided by Pharmacia Ophthalmics. Anders Marsk, MS assisted with study design and regulatory affairs and Thomas Kaponen, MS, of Pharmacia Ophthalmics assisted with the statistical analysis. 
1 Alm A, Villumsen J. PhXA34, a new potent ocular hypoten sive drug. A study on dose-response relationship and on aqueous humor dynamics in healthy volunteers. Arch Ophthalmol 1991; 109: 1564-8.

2 Villumsen J, Alm A. PhXA34 - a prostaglandin $\mathrm{F}_{2 \alpha}$ analogue. Effect on intraocular pressure in patients with ocular hypertension. Brf Ophthalmol 1992; 76: 214-7.

3 Camras CB, Schumer RA, Marsk A, Lustgarten JS, Serle JB, Stjernschantz J, et al. Intraocular pressure reduction with PhXA34, a new prostaglandin analogue, in patients with PhXA34, a new prostaglandin analogue, in patients with

4 Nagasubramanian S, Sheth Gp, Hitchings RA, Stjernschantz $\mathrm{J}$. Intraocular pressure-reducing effect of $\mathrm{PhXA41}$ in coular hypertension. Comparison of dose regimens. Ophthalmology 1993; 100: 1305-11.

5 Alm A, Villumsen J, Törnquist P, Mandahl A, Airaksinen J, Tuulonen $\mathrm{A}$, et al. Intraocular pressure-reducing effect of $\mathrm{PhXA41}$ in patients with increased eye pressure. A onemonth study. Ophthalmology 1993; 100: 1312-7.

6 Toris CB, Camras CB, Yablonski ME. Effects of PhXA41, a new prostaglandin $F_{2 \alpha}$ analog, on aqueous humor dynamics new prostaglandin $\mathrm{F}_{2 \alpha}$ analog, on aqueous humor dy
in human eyes. Ophthalmology 1993;100: 1297-304

7 Rácz P, Ruzsonyi MR, Nagy ZT, Bito LZ. Maintained intraocular pressure reduction with once-a-day application of a new prostaglandin $F_{2 \alpha}$ analogue (PhXA4l). An inhospital, placebo-controlled study. Arch Ophthalmol 1993; 111: 657-61.

8 Ziai N, Dolan JW, Kacere RD, Brubaker RF. The effects on aqueous dynamics of PhXA41, a new prostaglandin $F_{2 a}$ analogue, after topical application in normal and ocular hypertensive human eyes. Arch Ophthalmol 1993; 111: $1351-8$.

9 Friström B, Nilsson SEG. Interaction of PhXA41, a new prostaglandin analogue, with pilocarpine. A study on prostaglandin analogue, with pilocarpine. A study on patients with elevatec

10 Villumsen $\mathrm{J}, \mathrm{Alm} \mathrm{A}$. The effect of adding prostaglandin $\mathrm{F}_{2 \alpha}$ isopropylester to timolol in patients with open angle glaucoma. Arch Ophthalmol 1990; 108: 1102-5.
11 Lee PY, Shao H, Camras CB, Podos SM. Additivity of

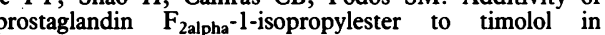
glaucoma patients. Ophthalmology 1991; 98: 1079-82.

12 Villumsen J. Effect of the prostaglandin $\mathrm{F}_{2}$ analogue $\mathrm{PhXA4}$ in human eyes treated with timolol and pilocarpine. In: Effects in human eyes of topical prostaglandin $\mathrm{F}_{2}$-isoproEffects in hus 17 eyes pylester and 17-phenyl substituted analogues of prostaglandin $F_{2 \alpha}$-isopropylester [Thesis]. Umeå: Um

13 Villumsen J, Alm A. Prostaglandin $F_{2 \alpha}$-isopropylester eye drops: effects in normal human eyes. Brf Ophthalmol 1989, 73: 419-26.

14 Selén G, Karlsson M, Astin M, Stjernschantz J, Resul B Effects of PhXA34 and PhDH100A, two phenyl substituted prostaglandin esters, on aqueous humor dynamics and microcirculation in the monkey eye. Invest Ophthalmol Vis Sci 1991; 32 (suppl): 869 (abstract).

15 Gabelt BT, Kaufman PL. The effect of prostaglandin $F_{2 \alpha}$ on trabecular outflow facility in cynomolgus monkeys. Exp Eye Res 1990; 51: 87-91.

16 Gabelt BT, Kaufman PL. Prostaglandin F $F_{2 a}$ increases uveoscleral outflow in the cynomolgus monkey. Exp Eye Res 1989; 49: 389-402.

17 Nilsson SFE, Samuelsson M, Bill A, Stjernschantz J. Increased uveoscleral outflow as a possible mechanism of ocular hypotension caused by prostaglandin $F_{2 \alpha}-1$-isopropylester in the cynomolgus monkey. Exp Eye Res 1989; 487: 707-16.

18 Kerstetter JR, Brubaker RF, Wilson SE, Kullerstrand LJ. Prostaglandin $F_{2 \alpha}$-1-isopropylester lowers intraocular pressure without decreasing aqueous humor flow. $A m \mathcal{F}$ Ophthalmol 1988; 105: 30-4.

19 Sjöquist B, Basu S, Resul B, Stjernschantz J. The pharmacokinetics of latanoprost in the cynomolgus monkey. Invest Cokinetics of latanoprost in the cynomolgus monkey.

20 Horie $\mathrm{T}$, Kitazawa $\mathrm{Y}$. The clinical significance of diurna variation in primary open-angle glaucoma. $f$ pn $\mathcal{F}$ Ophthalmol 1979; 23: 310-33. 